Published in final edited form as:

Hum Immunol. 2014 July ; 75(7): 614-620. doi:10.1016/j.humimm.2014.04.018.

\title{
CD40 expression in renal cell carcinoma is associated with tumor apoptosis, CD8+ T cell frequency and patient survival
}

\author{
Jonathan M. Weiss ${ }^{1}$, W. Gregory Alvord ${ }^{2}$, Octavio A. Quiñones ${ }^{2}$, Jimmy K. Stauffer ${ }^{1}$, and \\ Robert H. Wiltrout ${ }^{1}$ \\ ${ }^{1}$ Cancer and Inflammation Program, NCl, Center for Cancer Research, Frederick, MD \\ ${ }^{2}$ Statistical Consulting, Data Management Services, Inc., Frederick National Laboratory for \\ Cancer Research, Frederick, MD
}

\begin{abstract}
The co-stimulatory molecule, CD40, is expressed in renal cell carcinoma (RCC) and a variety of inflammatory diseases in the kidney. We investigated the relationship between tumor-associated CD40 expression, immune milieu of the tumor microenvironment, tumor stage and survival of patients with RCC. The expression of CD40, TUNEL and CD8 in human renal cell carcinomas was analyzed by immunohistochemistry performed on tissue samples obtained at the time of surgery. Computer-assisted quantitation of protein expression was used to analyze results in connection with patient survival and tumor stage. We show for the first time that tumor-associated CD40 expression is associated with prolonged survival in RCC patients. Tumor apoptosis (TUNEL) and CD8 immunostaining were also associated with patient survival. No relation was observed between CD40 expression and tumor stage. Our results suggest CD40 may be a prognostic biomarker indicative of prolonged RCC patient survival. Strategies that up-regulate CD40 expression in some RCC patients may thus improve survival, supporting further studies of agonistic CD40 antibodies in RCC.
\end{abstract}

\section{Keywords}

CD40; apoptosis; CD8; renal cell carcinoma; survival

\section{Introduction}

The co-stimulatory molecule CD40 is expressed by antigen-presenting cells (APCs) and plays a critical role in $\mathrm{T}$ cell activation and anti-cancer responses [1,2]. The triggering of CD40 on APCs via its ligand, CD154, is an important event in the production of cytokines [3] and mediation of immune responses against transformed cells $[4,1,2]$. CD40 is also widely expressed by non-hematopoietic cells [1,2]. In the kidney, it is expressed by tubular epithelial cells [5-8], endothelial cells [9,8], and mesangial cells [8]. Consistently, CD40

Corresponding Author: Jonathan M. Weiss, NCI Frederick, Bldg. 560, Rm. 31-18, Frederick, MD 21702, Ph (301) 846-5394, Fax (301) 846-1673,weissjo@mail.nih.gov.

The authors disclose no potential conflicts of interest. 
expression has been detected in association with renal cell carcinomas (RCC) [10,9] and upregulated expression has been noted in nephritis and other inflammatory renal diseases [8].

CD40 ligation on solid tumors transduces death signals via the induction of functional Fas ligand, Fas, TRAIL and TNF, as well as the activation of caspases 8 and 3, in apoptosissusceptible carcinoma cells [4]. We showed that CD40 is expressed by murine renal cancer cells and its ligation induced Fas expression that mediated tumor killing by Fas ligandpositive effector T cells [11]. We also found that CD40 ligation on human RCC triggers cytokine production, leukocyte recruitment and anti-tumor responses that were independent of host CD40 expression [12]. Stimulation of tumor-associated CD40 expression can therefore regulate microenvironment-specific immune responses and the susceptibility of tumor cells to cell death.

Due to the widespread expression of CD40 among diverse cell types, both normal and transformed, considerable effort has focused on ascertaining its role in regulating cell growth, proliferation, activation and host anti-tumor responses. Unfortunately, the prognostic value for CD40 expression by solid tumors is complex and may be dependent on the tumor type and the nature of $\mathrm{CD} 40^{+}$cells that predominate within the tumor microenvironment. CD40 expression is associated with clinical stage, degree of metastasis and poor prognosis in melanoma [13], gastric cancer [14], and non-small cell lung cancer [15]. In contrast, CD40, frequently expressed on tumor-associated macrophages, is associated with favorable prognosis in colorectal cancer [16], head and neck squamous cell carcinoma [17] and breast cancer [18]. Furthermore, CD40 ligation strongly inhibited breast cancer growth [19] and induced ovarian cancer [20] and colon cancer cell [21] apoptosis. Thus, the prognostic value for CD40 expression in RCC remains incompletely defined and may well relate to tumor type or the nature of other cells in close proximity to tumors.

Given the demonstrated abilities of CD40 to regulate immune and anti-tumor responses, we sought to determine whether there could be an association between CD40 expression and RCC patient outcome. Our data indicate that although CD40 expression was not related to RCC stage, its down-regulation was strongly associated with shortened patient survival. Moreover the levels of CD40 were associated with tumor cell apoptosis and CD8 ${ }^{+} \mathrm{T}$ cell infiltration. Our data indicate that expression of CD40, TUNEL and CD8 may serve as useful biomarkers for evaluating RCC patient prognosis, and may inform patient selection for certain immunotherapies. Given the ongoing development of agonistic and antagonistic CD40 antibodies in the clinic [22,23], our data further indicate that CD40-based targeting strategies may hold promise for treating patients with RCC.

\section{Materials and Methods}

\subsection{Source of human tissue samples.}

Kidney tissue microarrays were purchased from US Biomax, Inc. (Rockville, MD). For patient survival, the kidney cancer tissue array (Cat \# KD951) was used. For tumor grade and stage data, the renal disease spectrum tissue array (Cat \# KD2084) was used. Each slide consisted of duplicated cores and matched normal adjacent tissue. The samples were 
collected at the time of patient surgery. Patients had not received any therapy prior to surgery.

\subsection{Immunohistochemistry.}

Tissue microarray slides were deparaffinized with xylene and rehydrated through graded ethanols. Endogenous peroxidase was blocked with $0.6 \%$ hydrogen peroxide. Slides were blocked with Endogenous Biotin Block followed by normal horse serum (both from Vector Labs, Burlingame, CA). Negative control slides were incubated with normal mouse isotypecontrol antibody (BD Biosciences, San Jose, CA). For CD40 detection, slides were incubated with primary antibody [1:2000 dilution, overnight at $4{ }^{\circ} \mathrm{C}$, EMD Millipore (Billerica, MA)], biotinylated horse anti-mouse secondary antibody (1:100, Vector Labs), $\mathrm{ABC}$ reagent and DAB. CD8 immunostaining was performed on LeicaBiosystems' (Buffalo Grove, IL) Bond Autostainer with 10 min EDTA antigen retrieval, primary antibody [1:100 dilution, $30 \mathrm{~min}$ at RT, DAKO (Carpinteria, CA)], biotinylated secondary antibody and LeicaBiosystems' Intense R Detection Kit. Slides were counterstained with hematoxylin and cover-slipped.

For TUNEL assay, sections were treated with proteinase $\mathrm{K}$ for $15 \mathrm{~min}$ at RT following deparaffinization and rehydration of slides. After blocking endogenous peroxidase, slides were incubated with TdT (New England Biolabs, Ipswich, MA) $30 \mathrm{~min}$ at $37^{\circ} \mathrm{C}$. The reaction was detected with anti-digoxigenin-peroxidase (Roche Diagnostics Corp., Indianapolis, IN) and DAB.

In some cases, slides were co-incubated with anti-CD40 or TUNEL, along with antibody to CD45 (Dako), Von Willebrand Factor/Factor VIII (DAKO), Carbonic Anhydrase IX (Abcam, Cambridge, MA) or IL-12 p70 (Abcam). Immunoreactivity was visualized using $\mathrm{DAB}$ and alkaline phosphatase co-detection.

\subsection{Quantitation of Immunohistochemistry.}

Stained slides were analyzed on a Nikon Eclipse E400 microscope equipped with a Nikon DXM1200 digital camera and Nikon ACT-1 software (Melville, NY). The resulting images (100x magnification) were analyzed using Cell Profiler cell image analysis software [24]. For CD40, both the sum of pixel intensities of all objects and mean intensities were quantified. For TUNEL and CD8, the number of thresholded, stained objects was counted.

\subsection{Statistical analysis.}

The expression of CD40, TUNEL, and CD8 was evaluated with analysis of variance (anova) among three groups of interest: (1) Long-term survivors, (2) Short-term survivors and (3) Adjacent Normal. Post hoc Tukey tests were used to control for the Type I error rate in pairwise comparisons among these groups. CD40 expression values were also evaluated with respect to RCC subtypes and RCC tumor stage categories with analysis of variance (anova), and followed up with Dunnett's test to control for the Type I error rate in pairwise comparisons, taking Adjacent Normal as the reference group. Patient age and tumor size comparisons between long-term and short-term survivors were evaluated with t-tests. Follow-up survival times between long-term and short-term survivors were evaluated with 
the non-parametric Wilcoxon's Rank Sum test. Statistical computations were performed with the R Statistical Language and Environment [25]. All tests were two-sided; probability values $<0.05$ were considered significant.

\section{Results}

\subsection{Patient age and tumor size are not associated with survival.}

A total of 25 RCC cases which contained patient follow-up survival were analyzed. As shown in Table 1, 16 (64\%) of the patients had survived $>8$ years $(105.7 \pm 1.5$ months) following surgery. These patients are referred to as the "long-term survivors" group. Postsurgery survival follow-up was completed in 2011. Therefore, it remains possible, indeed likely, that surviving patients will experience ever increasing mean survival times. In contrast, 9 patients $(36 \%)$ died with a mean survival of $45.6 \pm 10.4$ months ( $<4$ years). These patients are referred to as the "short-term survivors" group. The cause of death for 8 of these 9 patients was cancer, and the cause of death for the remaining patient was chronic renal failure. With regards to the follow-up survival times, the difference between the longer- and shorter-term patient groups was highly significant $(\mathrm{p}<0.0001)$. However there was no significant difference in patient age or tumor size between the longer- and shorter-term survival patient groups.

\subsection{Higher CD40 expression in RCC is associated with patient survival.}

Immunohistochemistry of adjacent normal tissue indicated CD40 expression throughout the sample (Figure 1A). Strongest expression was detected in association with the vasculature, consistent with endothelial cell staining. Patients in the long-term survival stratum had a mean intensity CD40 expression of $377634 \pm 17621$. In contrast, patients in the short-term stratum had mean CD40 intensity expression of $290742 \pm 16596$. Among the longer surviving RCC patients, a relatively homogeneous and robust staining pattern was noted throughout the tumor tissue. In contrast, CD40 expression was weak to undetectable in the majority of cases among those patients who with shorter survival times. Differences in the sum of pixel intensities (Fig 1B) and mean intensity (Fig 1C) between the long-term surviving and dead patient groups were highly significant. As compared to adjacent normal controls, there was statistically significant decrease in CD40 expression within the tumor microenvironment of those patients who survived longer, which might be attributed in part to the presence of unstained, necrotic and cell-free zones in the tumor tissue.

\subsection{CD40 expression in RCC subtypes}

Next, we analyzed CD40 expression in a broad spectrum of renal diseases. As shown in Fig 2A, comparable CD40 immunostaining was detected in RCC and normal adjacent tissue. Similarly, no differences were observed in squamous cell carcinoma, papillary RCC, transitional cell carcinoma, metastatic RCC (isolated from adrenal gland, pelvic cavity or retroperitoneum) or nephritis (consisting of acute or chronic pyelonephritis and interstitial nephritis), as compared to normal adjacent controls. CD40 expression was, however, significantly down-regulated in Wilms' tumor, as compared to normal adjacent controls. 


\subsection{CD40 expression is not associated with stage of clear cell RCCs.}

We next further interrogated CD40 expression by stages of clear cell carcinomas (Figure 2B). No significant differences were detected between normal adjacent tissue and any stage of RCC

\subsection{The frequency of TUNEL+ and $\mathrm{CD}^{+}$cells is associated with RCC patient survival}

Since CD40 ligation on solid tumors has been associated with the induction of tumor cell apoptosis [4], we hypothesized that higher CD40 levels in the longer surviving patients could lead to increased apoptosis in these patients as well. Indeed, a significant increase in the number of TUNEL ${ }^{+}$cells within tumors was observed in long-term surviving patients, as compared to short-term surviving patients (Figures 3A and 3C).

CD40 ligation is associated with an induction of mononuclear cell recruitment and adhesion via increased chemokine and adhesion molecule expression in the kidney [6,7]. Furthermore, CD40 is a critical co-stimulatory molecule whose ligation can mediate the recruitment, expansion and activation of effector $\mathrm{CD} 8^{+} \mathrm{T}$ cells [26]. We therefore sought to determine whether $\mathrm{CD}^{+} \mathrm{T}$ cell frequency was altered between the $\mathrm{CD} 40$ high/longer surviving patients, as compared to the CD40 low/shorter-term surviving patient group. A significant increase in the number of $\mathrm{CD}^{+}$cells was observed in the long-term surviving patients' tumors (Figures 3B and 3D). In contrast, $\mathrm{CD}^{+}$cells were essentially undetectable among the normal adjacent tissue (Figure 3B). Although $\mathrm{CD} 8^{+}$cells were evident in the short-term surviving patients' samples, this was variable and not significantly different as compared to the normal adjacent controls.

\subsection{CD40 and TUNEL expression in long-term survivors are expressed primarily by tumor cells.}

Since CD40 and TUNEL expression were widespread throughout the tumor, we sought to determine which cells within the tumor microenvironment expressed CD40 and which were undergoing apoptosis. Using carbonic anhydrase IX (CAIX) as an RCC marker [27], we found widespread co-association of CD40 and CAIX expression in long-term surviving patients (Figure 4A). In contrast, CD40 reactivity within tumors was not associated with endothelial cells (Figure 4B). We also detected CD40-positive, MHC class II-positive antigen presenting cells (Figure 4C), as well as low but detectable IL-12 p70-positive cells (Figure 4D) in tumors, supporting the possibility that activated APCs also contribute to tumor-associated CD40 expression in tumors.

CAIX expression was also closely associated throughout the tumor tissue with TUNEL staining, further supporting the conclusion that the vast majority of apoptotic cells were tumor cells (Figure 4E). Although we cannot exclude the possibility that endothelial cells or leukocytes may be undergoing apoptosis, the majority of TUNEL staining was not colocalized to either endothelial (Figure 4F) or leukocyte (Figure 4G) cell staining. No appreciable difference in Factor VIII, MHC class II, IL-12 p70 or CD45 expression was noted between long-term and short-term surviving patients (data not shown). Taken together, these data suggest tumor cells represent the majority of CD40- and TUNEL-positive cells within patient samples. Furthermore, the co-association of up-regulated CD40 expression 
with increased frequency of tumor apoptosis corresponded to increased $\mathrm{CD} 8^{+} \mathrm{T}$ cell frequency within RCC tumors.

\section{Discussion}

In this study, we demonstrate the prognostic value for CD40 expression in human RCC.

Since clear cell morphology predominates in a majority of RCC patients, we focused on this tumor subtype and show for the first time the combined up-regulation of CD40, TUNEL and CD8 expression in patients with significantly longer survival ( $>8$ years), as compared to poorer survival ( $<4$ years). In one earlier study, Bussolati, et al [10] analyzed RCC cell lines and showed no correlation existed between CD40 expression and tumor stage, a finding we corroborated in clear cell RCC samples. We also show CD40 expression was up-regulated in metastatic RCC, down-regulated in Wilms' tumor and transitional cell carcinoma and relatively unchanged among the remaining spectra of kidney diseases we interrogated. To our knowledge, ours is the first study to demonstrate the prognostic significance of CD40 expression directly in RCC tumors.

CD40 is an attractive therapeutic target by virtue of its ability to mediate essential APC stimulation [1,3] and provision of help for memory $\mathrm{CD} 8^{+} \mathrm{T}$ cells [26]. Although we cannot rule out potentially important contributions by tumor-infiltrating hematopoietic cells, our immunohistochemical results demonstrated the majority of CD40 expression was tumorassociated, consistent with our previous demonstrations of CD40 expression by murine and human RCC [11,12]. On certain CD40 ${ }^{+}$solid tumors, CD40 ligation mediates apoptosis and direct anti-tumor responses [4,28], along with leukocyte recruitment [1], in at least a partially hematopoietic cell-independent manner [12]. Based on the ability of agonistic CD40 or CD40 ligand antibodies to stimulate immune responses and target tumors, CD40/ CD40L targeting reagents were identified as having great promise for use in cancer therapy by the Translational Research Acceleration Initiative [29].

We demonstrate the positive association between $\mathrm{CD} 40$ and $\mathrm{CD} 8^{+} \mathrm{T}$ cell infiltration in RCC. Among tumor types, RCC exhibits a relatively high degree of sensitivity to adoptive immunotherapy with lymphokine-activated killer and cytotoxic $\mathrm{T}$ cells $[30,31]$ and tumorinfiltrating lymphocytes have been shown to lyse autologous tumor cells [32]. CD8 ${ }^{+} \mathrm{T}$ cell frequency in RCC can be an important predictor of improved patient prognosis [33-35], however its use as a prognostic factor in the immune monitoring of RCC patients with combined immunotherapy is surprisingly complex $[36,33,37,38]$. We also show that CD40 expression and tumor cell apoptosis co-associate among surviving RCC patients which is consistent with the fact that CD40 ligation directly mediates apoptosis in carcinoma cells $[4,28]$. Overexpression of anti-apoptotic factors in RCC and a variety of solid tumors is frequently observed $[39,40]$ and associated with chemo- or radio-resistance and failure of many therapies $[41,42]$. CD40-mediated induction of apoptosis provides a mechanistic basis for the association between CD40 and improved patient outcome and underscores the attractiveness of CD40-based therapies for RCC.

Carbonic anhydrase IX (CAIX) is another well-studied prognostic factor for RCC. CAIX expression is ubiquitous in clear cell RCC because it is regulated by the Von Hippel Lindau 
(VHL) protein which is invariably lost in these tumors. CAIX expression was originally shown to associate with tumor grade [27] and CAIX overexpression correlated with better prognosis in patients receiving cytokines or new targeted drugs [43,44]. By ELISA, serum CAIX levels were related to tumor size in RCC patients [45]. A long-term follow-up study, however, found CAIX was not an independent prognostic marker for RCC [46]. In contrast to CAIX, CD40 expression was associated with long-term survival, but not with tumor stage or size. CD40 may therefore be a more selective indicator for predicting patients' anti-tumor responses.

Although we observed statistically significant differences, a limitation of our study is that we had access to a relatively small sample size of cases. Future studies with larger sample numbers may allow extrapolation of our results to more patients and address more complex questions relating to association between immune responses and RCC patient prognosis. Specifically, what is the basis for up-regulated CD40 expression in surviving RCC patients? Is tumor-associated CD40 increased by host immunologic factors, such as heightened cytokine expression, which may be associated with a nascent anti-tumor response, in surviving patients? Do tumor-intrinsic factors (e.g. mutation) regulate CD40 expression levels and lead to the development of heterogeneity with respect to tumor sensitivity to CD40-mediated apoptosis? Does CD40 ligation induce apoptosis in human RCC as we have shown for murine tumors [11]? It is also unknown whether increased CD40 expression triggers the release of chemokines capable of recruiting $\mathrm{CD} 8^{+} \mathrm{T}$ cells or whether activated $\mathrm{CD}^{+} \mathrm{T}$ cells produce cytokines (e.g. IFN $\gamma$ ) that up-regulate $\mathrm{CD} 40$ expression in the tumor microenvironment. Irrespective of the sequence of events, is the co-association of CD40 with TUNEL and CD8 in RCC tissues part of a mechanism for improving survival?

Although our findings do not permit definitive assignment of CD40 in mediating anti-tumor responses in RCC, they do indicate its expression levels may be a useful biomarker of patient survival. The reciprocal regulation of CD40 by cytokines (e.g. TNF, IFN $\gamma$ ) and Toll receptor activation (e.g. $\mathrm{CpG}$ ) is well studied $[3,47]$ and we hypothesize that such cytokine or DC-stimulating therapeutic approaches up-regulate tumor-associated CD40 expression which could be an important component of any survival improvement achieved by the therapy. We believe our data supports the possibility of screening RCC surgical material on the basis of CD40 expression for patient stratification for immunotherapeutic or other protocols. Specifically, agonistic CD40 antibodies, in particular, may have considerable value for the treatment of RCC. As more clinical material becomes available, it would be extremely useful to investigate the degree to which patient responsiveness to particular immunotherapies (e.g agonistic CD40 antibody, IL-2, IFN) may be related to tumorassociated CD40 expression.

\section{Acknowledgements}

This project was funded in whole or part by the Intramural Research Program of NIH/NCI. We thank Donna Butcher of the Pathology/Histotechnology Laboratory (NCI) for immunohistochemical staining.

\section{Abbreviations:}

TUNEL Terminal deoxynucleotidyl transferase dUTP nick end labeling 

RCC
renal cell carcinoma
APC
antigen presenting cells
CTL
cytotoxic T lymphocyte

\section{References}

1. Grewal IS, Flavell RA. CD40 and CD154 in cell-mediated immunity. Annu Rev Immunol 1998; 16:111-35. [PubMed: 9597126]

2. van Kooten C, Banchereau J. CD40-CD40 ligand. J Leukoc Biol 2000; 67 (1):2-17. [PubMed: 10647992]

3. Schulz O, Edwards AD, Schito M, Aliberti J, Manickasingham S, Sher A et al. CD40 triggering of heterodimeric IL-12 p70 production by dendritic cells in vivo requires a microbial priming signal. Immunity 2000; 13 (4):453-62. [PubMed: 11070164]

4. Eliopoulos AG, Davies C, Knox PG, Gallagher NJ, Afford SC, Adams DH et al. CD40 induces apoptosis in carcinoma cells through activation of cytotoxic ligands of the tumor necrosis factor superfamily. Mol Cell Biol 2000; 20 (15):5503-15. [PubMed: 10891490]

5. Laxmanan S, Datta D, Geehan C, Briscoe DM, Pal S. CD40: a mediator of pro- and antiinflammatory signals in renal tubular epithelial cells. J Am Soc Nephrol 2005; 16 (9):2714-23. [PubMed: 16033859]

6. Li H, Nord EP. CD40 ligation stimulates MCP-1 and IL-8 production, TRAF6 recruitment, and MAPK activation in proximal tubule cells. Am J Physiol Renal Physiol 2002; 282 (6):F1020-33. [PubMed: 11997318]

7. Li H, Nord EP. CD40/CD154 ligation induces mononuclear cell adhesion to human renal proximal tubule cells via increased ICAM-1 expression. Am J Physiol Renal Physiol 2005; 289 (1):F145-53. [PubMed: 15713910]

8. Yellin MJ, D'Agati V, Parkinson G, Han AS, Szema A, Baum D et al. Immunohistologic analysis of renal CD40 and CD40L expression in lupus nephritis and other glomerulonephritides. Arthritis Rheum 1997; 40 (1):124-34. [PubMed: 9008608]

9. Kluth B, Hess S, Engelmann H, Schafnitzel S, Riethmuller G, Feucht HE. Endothelial expression of CD40 in renal cell carcinoma. Cancer Res 1997; 57 (5):891-9. [PubMed: 9041191]

10. Bussolati B, Russo S, Deambrosis I, Cantaluppi V, Volpe A, Ferrando U et al. Expression of CD154 on renal cell carcinomas and effect on cell proliferation, motility and platelet-activating factor synthesis. Int J Cancer 2002; 100 (6):654-61. [PubMed: 12209602]

11. Lee JK, Seki N, Sayers TJ, Subleski J, Gruys EM, Murphy WJ et al. Constitutive expression of functional CD40 on mouse renal cancer cells: induction of Fas and Fas-mediated killing by CD40L. Cell Immunol 2005; 235 (2):145-52. [PubMed: 16213477]

12. Shorts L, Weiss JM, Lee JK, Welniak LA, Subleski J, Back T et al. Stimulation through CD40 on mouse and human renal cell carcinomas triggers cytokine production, leukocyte recruitment, and antitumor responses that can be independent of host CD40 expression. J Immunol 2006; 176 (11): 6543-52. [PubMed: 16709811]

13. van den Oord JJ, Maes A, Stas M, Nuyts J, Battocchio S, Kasran A et al. CD40 is a prognostic marker in primary cutaneous malignant melanoma. Am J Pathol 1996; 149 (6):1953-61. [PubMed: 8952530]

14. Li R, Chen WC, Pang XQ, Hua C, Li L, Zhang XG. Expression of CD40 and CD40L in gastric cancer tissue and its clinical significance. Int J Mol Sci 2009; 10 (9):3900-17. [PubMed: 19865524]

15. Ishikawa K, Miyamoto M, Yoshioka T, Kato T, Kaji M, Ohbuchi T et al. Up-regulation of CD40 with juxtacrine activity in human nonsmall lung cancer cells correlates with poor prognosis. Cancer 2008; 113 (3):530-41. [PubMed: 18548529]

16. Kinouchi M, Miura K, Mizoi T, Ishida K, Fujibuchi W, Sasaki H et al. Infiltration of CD40Positive Tumor-Associated Macrophages Indicates a Favorable Prognosis in Colorectal Cancer Patients. Hepatogastroenterology 2012; 60 (121). 
17. Sathawane D, Kharat RS, Halder S, Roy S, Swami R, Patel R et al. Monocyte CD40 expression in head and neck squamous cell carcinoma (HNSCC). Hum Immunol 2013; 74 (1):1-5. [PubMed: 23000501]

18. Slobodova Z, Ehrmann J, Krejci V, Zapletalova J, Melichar B. Analysis of CD40 expression in breast cancer and its relation to clinicopathological characteristics. Neoplasma 2011; 58 (3):18997. [PubMed: 21391734]

19. Wingett DG, Vestal RE, Forcier K, Hadjokas N, Nielson CP. CD40 is functionally expressed on human breast carcinomas: variable inducibility by cytokines and enhancement of Fas-mediated apoptosis. Breast Cancer Res Treat 1998; 50 (1):27-36. [PubMed: 9802617]

20. Zhou Y, He J, Gou LT, Mu B, Liao WC, Ma C et al. Expression of CD40 and growth-inhibitory activity of CD40 agonist in ovarian carcinoma cells. Cancer Immunol Immunother 2012; 61 (10): 1735-43. [PubMed: 22406982]

21. Wu Y, Wang L, He X, Xu H, Zhou L, Zhao F et al. Expression of CD40 and growth-inhibitory activity of CD40 ligand in colon cancer ex vivo. Cell Immunol 2008; 253 (1-2):102-9. [PubMed: 18603231]

22. Vonderheide RH. Prospect of targeting the CD40 pathway for cancer therapy. Clin Cancer Res 2007; 13 (4):1083-8. [PubMed: 17317815]

23. Vonderheide RH, Glennie MJ. Agonistic CD40 antibodies and cancer therapy. Clin Cancer Res 2013; 19 (5):1035-43. [PubMed: 23460534]

24. Carpenter AE, Jones TR, Lamprecht MR, Clarke C, Kang IH, Friman O et al. CellProfiler: image analysis software for identifying and quantifying cell phenotypes. Genome Biol 2006; 7 (10):R100. [PubMed: 17076895]

25. Team RC (2013) R: A language and environment for statistical computing. R Foundation for Statistical Computing http://www.R-project.org/.

26. Shugart JA, Bambina S, Alice AF, Montler R, Bahjat KS. A Self-Help Program for Memory CD8+ T Cells: Positive Feedback via CD40-CD40L Signaling as a Critical Determinant of Secondary Expansion. PLoS One 2013; 8 (5):e64878. [PubMed: 23717671]

27. Genega EM, Ghebremichael M, Najarian R, Fu Y, Wang Y, Argani P et al. Carbonic anhydrase IX expression in renal neoplasms: correlation with tumor type and grade. Am J Clin Pathol 2010; 134 (6):873-9. [PubMed: 21088149]

28. Gallagher NJ, Eliopoulos AG, Agathangelo A, Oates J, Crocker J, Young LS. CD40 activation in epithelial ovarian carcinoma cells modulates growth, apoptosis, and cytokine secretion. Mol Pathol 2002; 55 (2):110-20. [PubMed: 11950960]

29. Cheever MA. Twelve immunotherapy drugs that could cure cancers. Immunol Rev 2008; 222:35768. [PubMed: 18364014]

30. Thompson JA, Figlin RA, Sifri-Steele C, Berenson RJ, Frohlich MW. A phase I trial of CD3/ CD28-activated T cells (Xcellerated T cells) and interleukin-2 in patients with metastatic renal cell carcinoma. Clin Cancer Res 2003; 9 (10 Pt 1):3562-70. [PubMed: 14506142]

31. Rosenberg SA, Lotze MT, Muul LM, Chang AE, Avis FP, Leitman S et al. A progress report on the treatment of 157 patients with advanced cancer using lymphokine-activated killer cells and interleukin-2 or high-dose interleukin-2 alone. N Engl J Med 1987; 316 (15):889-97. [PubMed: 3493432]

32. Belldegrun A, Muul LM, Rosenberg SA. Interleukin 2 expanded tumor-infiltrating lymphocytes in human renal cell cancer: isolation, characterization, and antitumor activity. Cancer Res 1988; 48 (1):206-14. [PubMed: 3257161]

33. Arima K, Nakagawa M, Yanagawa M, Sugimura Y, Tochigi H, Kawamura J. Prognostic factors of peripheral blood lymphocyte subsets in patients with renal cell carcinoma. Urol Int 1996; 57 (1):510. [PubMed: 8840483]

34. Tomita Y, Nishiyama T, Fujiwara M, Sato S. Immunohistochemical detection of major histocompatibility complex antigens and quantitative analysis of tumour-infiltrating mononuclear cells in renal cell cancer. Br J Cancer 1990; 62 (3):354-9. [PubMed: 2206942]

35. Kondo T, Ito F, Nakazawa H, Horita S, Osaka Y, Toma H. High expression of chemokine gene as a favorable prognostic factor in renal cell carcinoma. J Urol 2004; 171 (6 Pt 1):2171-5. [PubMed: 15126779] 
36. Gohring B, Riemann D, Rebmann U, Heynemann H, Schabel J, Langner J. Prognostic value of the immunomonitoring of patients with renal cell carcinoma under therapy with IL-2/IFN-alpha-2 in combination with 5-FU. Urol Res 1996; 24 (5):297-303. [PubMed: 8931295]

37. Characiejus D, Pasukoniene V, Kazlauskaite N, Valuckas KP, Petraitis T, Mauricas M et al. Predictive value of CD8highCD57+ lymphocyte subset in interferon therapy of patients with renal cell carcinoma. Anticancer Res 2002; 22 (6B):3679-83. [PubMed: 12552976]

38. Hernberg M, Muhonen T, Pyrhonen S. Can the CD4+/CD8+ ratio predict the outcome of interferon-alpha therapy for renal cell carcinoma? Ann Oncol 1997; 8 (1):71-7.

39. Lei Y, Geng Z, Guo-Jun W, He W, Jian-Lin Y. Prognostic significance of survivin expression in renal cell cancer and its correlation with radioresistance. Mol Cell Biochem 2010; 344 (1-2):2331. [PubMed: 20563741]

40. LaCasse EC, Baird S, Korneluk RG, MacKenzie AE. The inhibitors of apoptosis (IAPs) and their emerging role in cancer. Oncogene 1998; 17 (25):3247-59. [PubMed: 9916987]

41. Yuan QZ, Wang CT, Mao YQ, Zhang P, Shi HS, Li ZY et al. Enhanced tumor radiosensitivity by a survivin dominant-negative mutant. Oncol Rep 2010; 23 (1):97-103. [PubMed: 19956869]

42. Virrey JJ, Guan S, Li W, Schonthal AH, Chen TC, Hofman FM. Increased survivin expression confers chemoresistance to tumor-associated endothelial cells. Am J Pathol 2008; 173 (2):575-85. [PubMed: 18599610]

43. Muriel Lopez C, Esteban E, Astudillo A, Pardo P, Berros JP, Izquierdo M et al. Predictive factors for response to treatment in patients with advanced renal cell carcinoma. Invest New Drugs 2012; 30 (6):2443-9. [PubMed: 22644070]

44. Stillebroer AB, Mulders PF, Boerman OC, Oyen WJ, Oosterwijk E. Carbonic anhydrase IX in renal cell carcinoma: implications for prognosis, diagnosis, and therapy. Eur Urol 2010; 58 (1):7583. [PubMed: 20359812]

45. Zhou GX, Ireland J, Rayman P, Finke J, Zhou M. Quantification of carbonic anhydrase IX expression in serum and tissue of renal cell carcinoma patients using enzyme-linked immunosorbent assay: prognostic and diagnostic potentials. Urology 2010; 75 (2):257-61. [PubMed: 19963243]

46. Zhang BY, Thompson RH, Lohse CM, Dronca RS, Cheville JC, Kwon ED et al. Carbonic anhydrase IX (CAIX) is not an independent predictor of outcome in patients with clear cell renal cell carcinoma (ccRCC) after long-term follow-up. BJU Int 2013; 111 (7):1046-53. [PubMed: 23551810]

47. Buhtoiarov IN, Lum HD, Berke G, Sondel PM, Rakhmilevich AL. Synergistic activation of macrophages via CD40 and TLR9 results in T cell independent antitumor effects. J Immunol 2006; 176 (1):309-18. [PubMed: 16365423] 
A) Long-term survivor

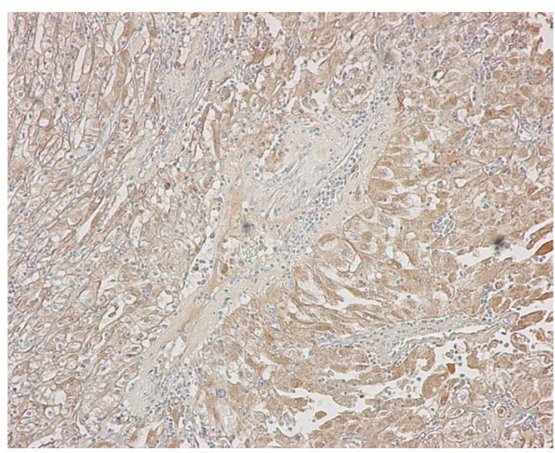

Short-term survivor

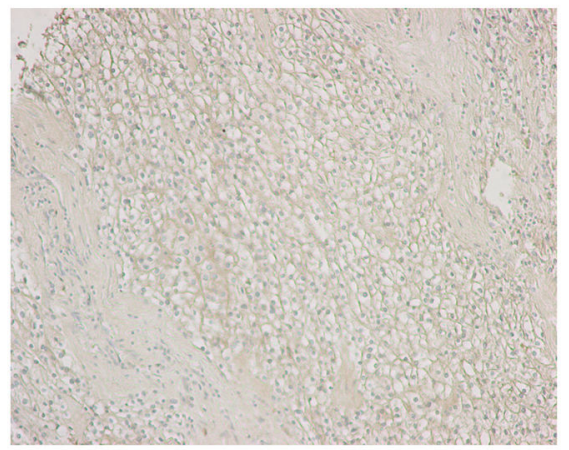

Adjacent normal

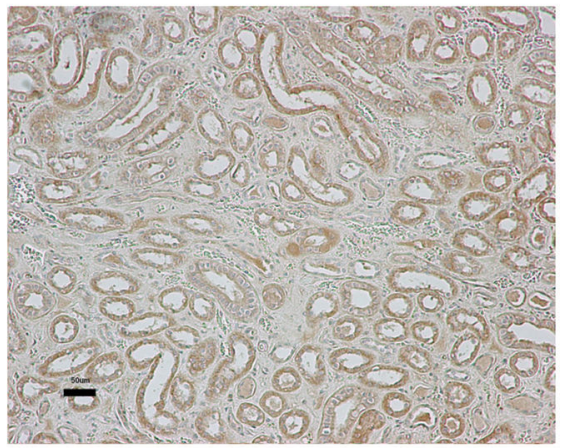

B)

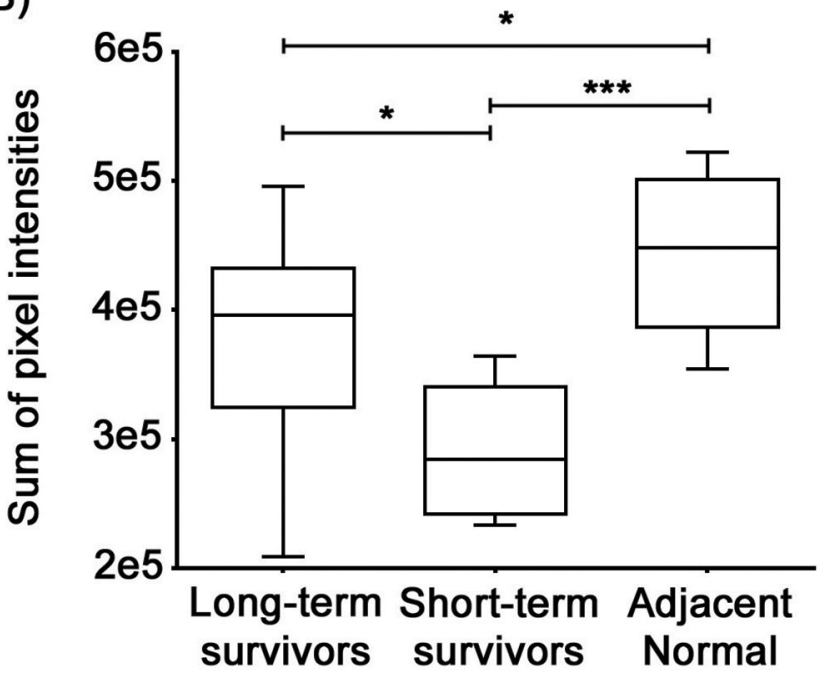

C)

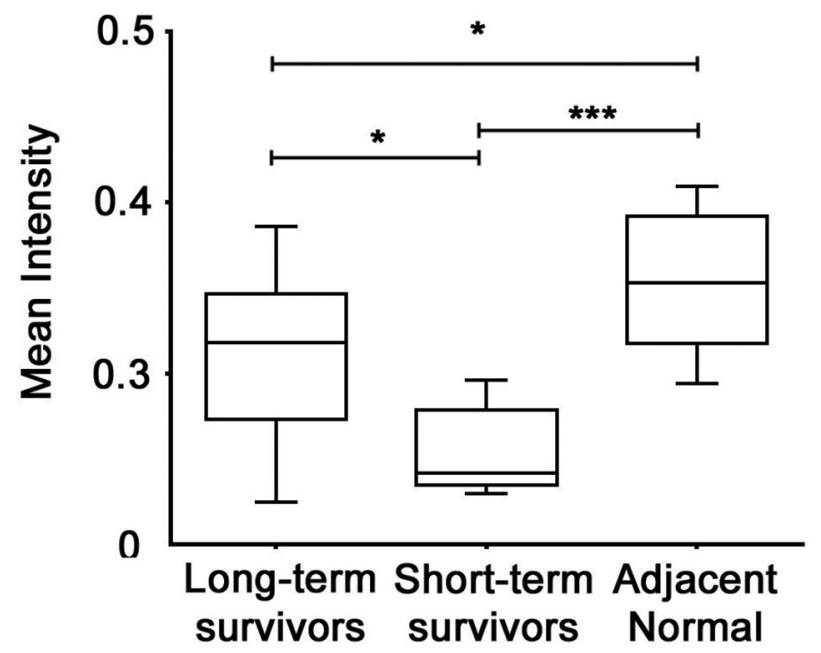

Figure 1. CD40 expression is associated with RCC patient survival.

(A) Clear cell RCC cases were analyzed for CD40 expression by immunohistochemistry. Adjacent normal tissue was used as comparison. Results are representative of 16 longer surviving, 9 shorter surviving and 9 adjacent normal control cases. Immunostaining was quantitated using Cell Profiler and graphed as either the (B) sum of pixel intensities or (C) mean intensity of brown DAB staining. (* $\mathrm{p}<0.05$; *** $\mathrm{p}<0.0005)$. 
A) B)

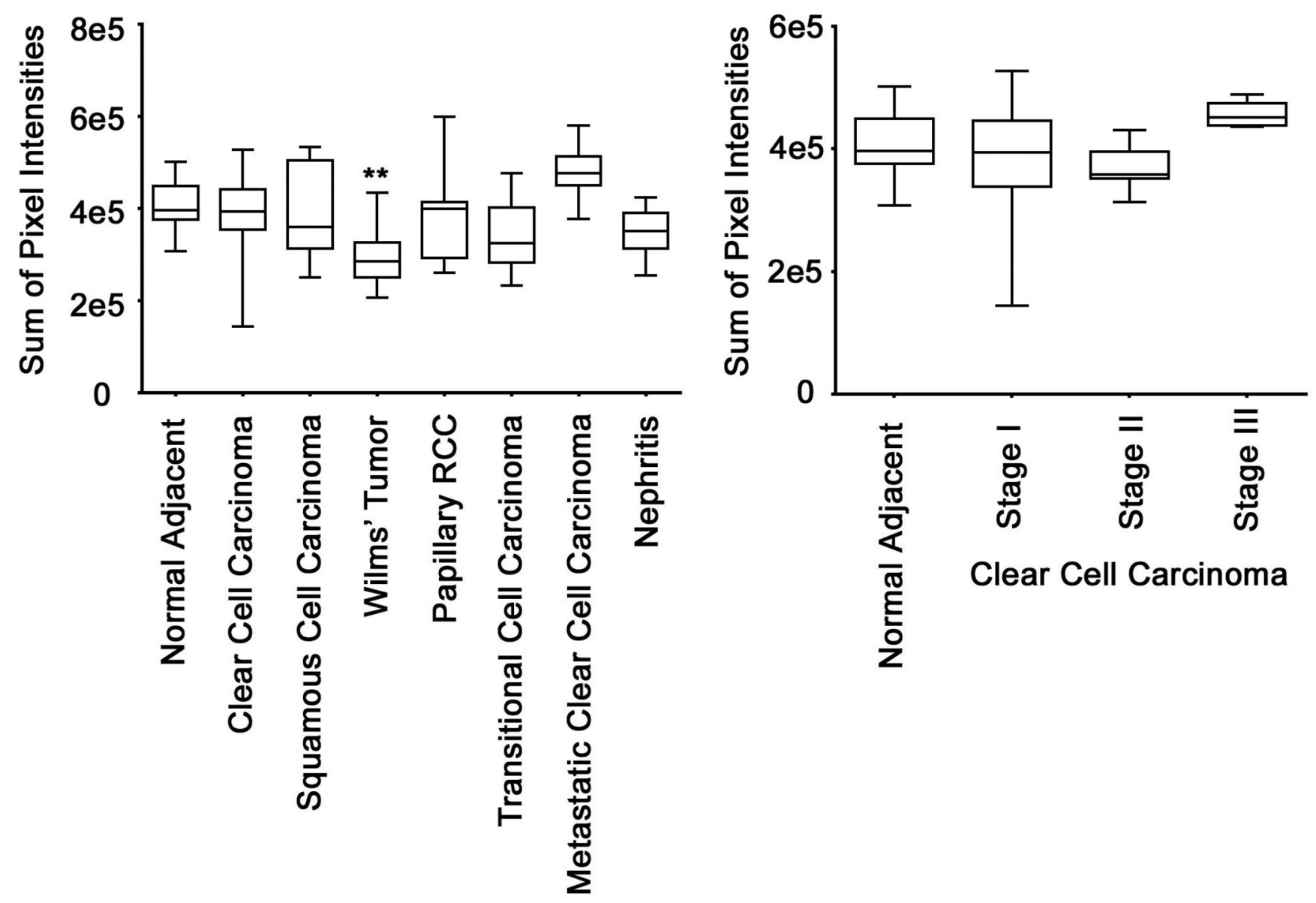

Figure 2. CD40 expression in RCC subtypes and RCC tumor stages.

The sums of CD40 immunohistochemistry staining intensities for each patient were analyzed in the renal disease spectrum tissue array. (A) Results are derived from duplicate cores (fields) each of 30 cases of clear cell carcinoma, 5 cases of squamous cell carcinoma, 10 cases of Wilms' tumor, 10 cases of papillary RCC, 18 cases of transitional cell carcinoma, 4 cases of metastatic clear cell carcinoma and 5 cases of nephritis. Statistical significance was compared to the normal adjacent controls $(* * \mathrm{p}<0.005)$. (B) The 30 cases of clear cell carcinoma were subdivided based upon the provided tumor stage and TNM information. There were 21 cases of stage I, 6 cases of stage II and 3 cases of stage III RCC. 


\section{A) TUNEL}
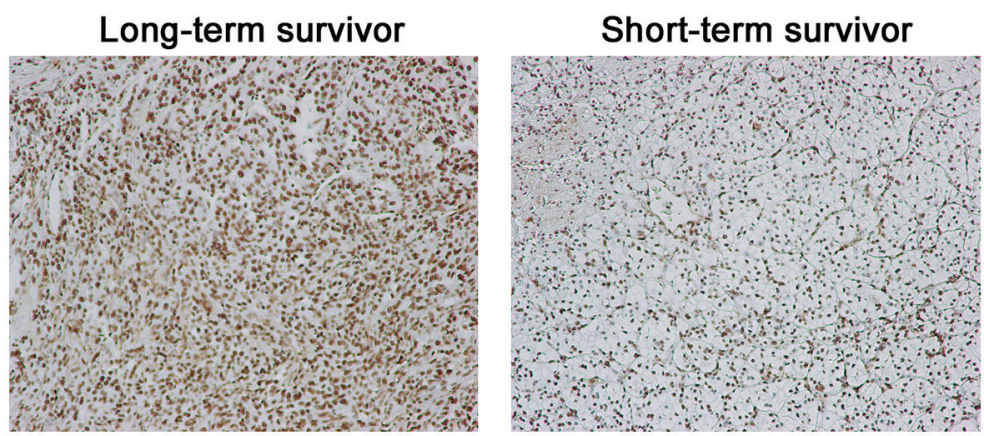

B) CD8

Long-term survivor

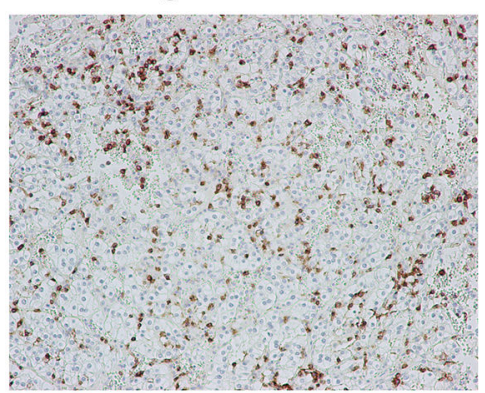

Short-term survivor

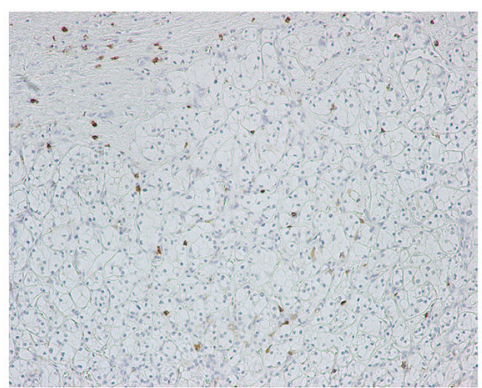

Adjacent normal

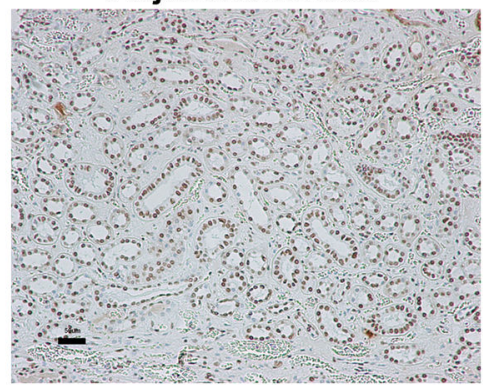

Adjacent normal

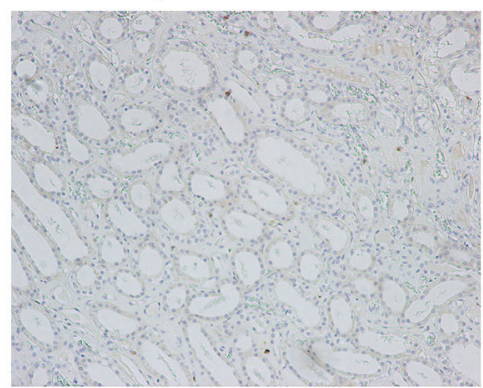

C)

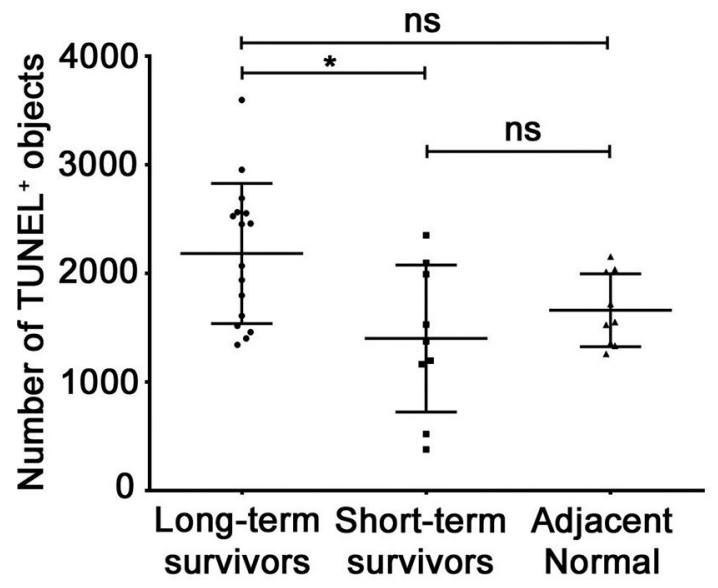

D)

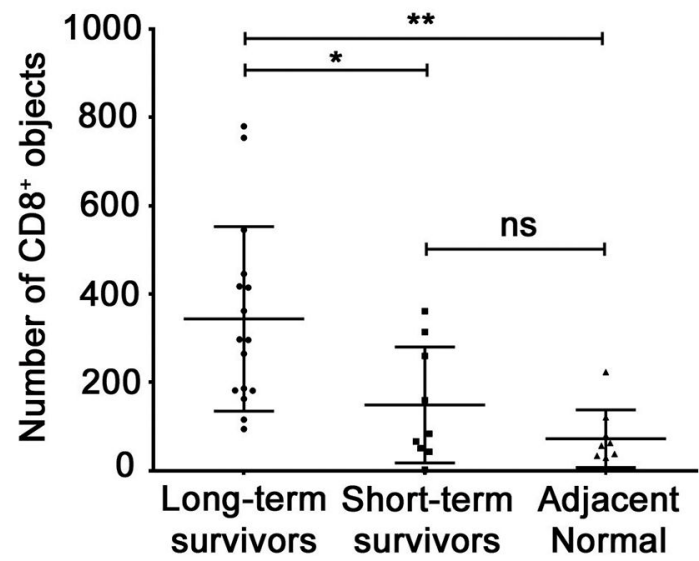

Figure 3. TUNEL and CD8 staining is associated with RCC patient survival.

Clear cell RCC cases were analyzed for (A) TUNEL and (B) CD8 expression by immunohistochemistry and compared to adjacent normal controls. Each data point on the graph is derived from the average quantitated value of 2 non-overlapping fields per patient. Results are representative of 16 longer surviving, 9 shorter surviving and 9 adjacent normal control cases. The number of (C) TUNEL and (D) CD8 positive objects was quantified using Cell Profiler $(* \mathrm{p}<0.05 ; * * \mathrm{p}<0.005 ; \mathrm{ns}=$ not significant for the indicated comparisons $)$. 
A) CD40 / CAIX

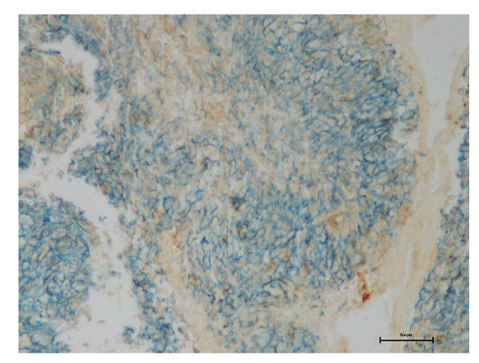

C) CD40 / MHC Class II

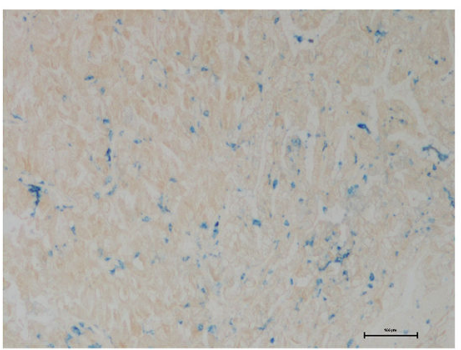

E) TUNEL / CAIX

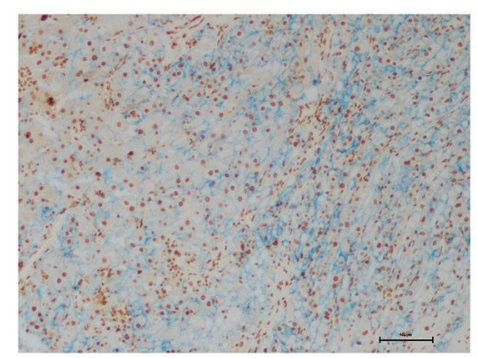

G) TUNEL / CD45
B) CD40 / Factor VIII

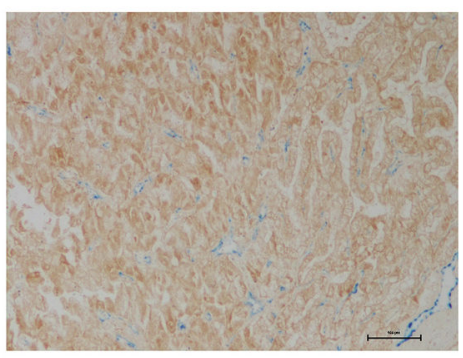

D) CD40 / IL-12 p70

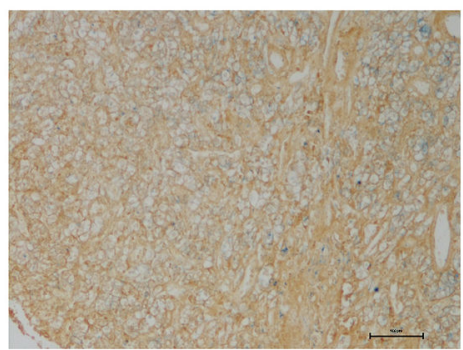

F) TUNEL / Factor VIII

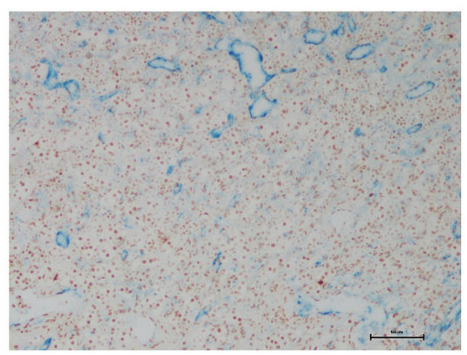

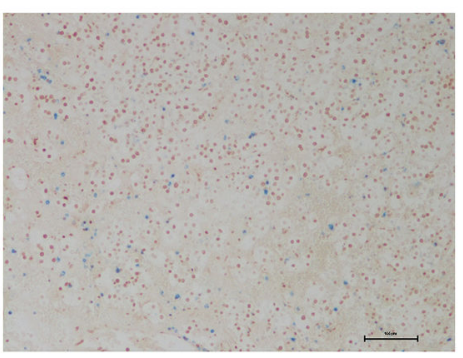

Figure 4. Tumor cells express the majority of CD40 and TUNEL expression.

Clear cell RCC cases were analyzed by immunohistochemistry for the indicated markers. A representative long-term surviving patient sample is shown. CD40 (A-D) and TUNEL (E-G) reactivity was visualized by brown DAB staining. CAIX, Factor VIII, MHC class II, IL-12 p70 and CD40 reactivity was visualized using blue alkaline phosphatase staining. Scale bar $=100 \mu \mathrm{M}$. 
Table 1.

Patient Characteristics.

25 patients with clear cell RCC were stratified into two groups, based upon their follow-up survival status. The means ( \pm standard error) for patient age, primary tumor size and follow-up survival times are shown for each patient group.

\begin{tabular}{|c|c|c|c|}
\hline & Long-term survivors $(\mathbf{n = 1 6})$ & Short-term survivors $(\mathbf{n = 9})$ & P value \\
\hline Patient age (years) & $53.8 \pm 2.1$ & $59.2 \pm 4.4$ & 0.217 \\
\hline Tumor size $\left(\mathrm{cm}^{2}\right)$ & $6.3 \pm 0.6$ & $8.1 \pm 1.4$ & 0.167 \\
\hline Follow-up (months) & $105.7 \pm 1.5$ & $45.6 \pm 10.4$ & $<0.0001$ \\
\hline
\end{tabular}

